\title{
Electron Beam Heating of Water Droplets During in-situ ESEM Imaging of Condensation on Superhydrophobic Surfaces
}

\author{
K. Rykaczewski ${ }^{*}$, J.H.J. Scott*, and A. G. Fedorov ${ }^{* *}$ \\ * Material Measurement Laboratory, National Institute of Standards and Technology, Gaithersburg, \\ MD 20899 \\ ** W. Woodruff School of Mechanical Engineering, Georgia Institute of Technology, Atlanta, GA \\ 30332
}

Vapor condensation is an essential part of many technologies in energy generation and even a moderate increase in the heat transfer efficiency during this phase change process could lead to considerable economic savings. The heat transfer rate can be increased by an order of magnitude by promoting dropwise, rather than the commonly occurring filmwise, condensation [1]. However, premature degradation of the surfaces and coatings needed to maintain dropwise condensation has prevented any practical application of the process [2]. Due to the lotus flower like ability of superhydrophobic surfaces (SHS) to shed water, a lot of research effort has been dedicated to their application as promoters for dropwise condensation. Several groups have demonstrated that SHS with properly designed nanoscale topology can maintain dropwise condensation [3,4]. Most of the research effort in this area has been dedicated to the fabrication of the SHS themselves and characterization of their wetting behavior [5]. The few studies that focused on the condensation dynamics [6,7] restricted the range of diameters of studied drops to $\sim 10 \mu \mathrm{m}$ to a few millimeters. However, we have previously demonstrated that during condensation on a nanostructured SHS the number of droplets with diameters below $10 \mu \mathrm{m}$ is significantly increased as compared to condensation on flat hydrophobic surface [8]. Furthermore, the sub-10 $\mu \mathrm{m}$ droplets account for the majority of the heat transferred during dropwise condensation [9]. Because of the small size of the droplets, the dynamic nature of the phase change process, and the complex topography of the SHS, in-situ Environmental Scanning Electron Microscopy (ESEM) is the preferred method for imaging the growth of droplets in the sub-10 $\mu \mathrm{m}$ regime. In this work, we demonstrate that during high magnification ESEM imaging of condensation on cupric hydroxide nanotube SHS the electron beam can cause significant heating and fast evaporation of the condensed droplets. As shown in Figure 1a, we characterize the electron beam heating effects by observing the evaporation rates of condensed water droplets under different conditions in the ESEM. We quantify those effects by evaluating the characteristic length, $L^{*}$, defined as four times the ratio of the drop area divided by the perimeter, for each captured image. For all imaging settings the decrease of $L *$ is linear in time, allowing for the use of calculated $d L * / d t$ as a criterion for comparison of the electron beam heating effect at different beam energies, currents, and viewing areas. As shown in Figure b, $d L * / d t$ increases with both the electron beam flux (current/area) and energy. These experimental trends agree well with predictions of single energy deposition/conduction model [10]. Based on our results, at optimal ESEM imaging settings ( $10 \mathrm{keV}$ and $0.013 \mathrm{nA}$ ), electron beam heating effects limit the viewing area to $\sim 4.6 \mu \mathrm{m}$ by $\sim 4.3 \mu \mathrm{m}$. As shown in Figure 1c, when imaged using those settings individual water droplet growth proceeds by first wetting of an area with $\sim 2 \mu \mathrm{m}-4 \mu \mathrm{m}$ characteristic dimension between the nanotubes, then growing in constant base area mode until reaching a nearly spherical shape with diameter of $\sim 4 \mu \mathrm{m}-6 \mu \mathrm{m}$, and lastly growing in constant contact angle mode until combining with other drops [11]. 


\section{References}

[1] E. Schmidt, et al., Forschung im Ingenieurwesen 1 (2) (1930) 53-63.

[2] V. P. Carey, Liquid-Vapor Phase-Change Phenomena, $2^{\text {nd }}$ ed., Taylor\&Francis,New York, 2008.

[3] K. K. S. Lau, et al., Nano Lett. 3 (2003) 1701-1705.

[4] C. H. Chen, et al., App. Phys. Lett. 90 (2007) 3.

[5] C. Dorrer and J. Ruhe, Soft Matter. 5 (2009) 51-61.

[6] M. Nosonovsky and B. Bhushan, Nano Lett. 7 (2007) 2633-2637.

[7] Y. M. Zheng, et al. Appl. Phys. Lett. 92 (2008) 3.

[8] C. Dietz, et al., App. Phys. Lett. 97 (2010) 033104-1.

[9] C. Graham and P. Griffith, Int. J. Heat Mass Transfer 16 (1973) 337-346.

[10] K. Rykaczewski et al., Appl. Phys. Lett. (2011), in press.

[11] This research was performed while K. Rykaczewski held a NRC ARRA Fellowship the NIST in Gaithersburg, MD. A. G. Fedorov was supported by AFOSR BIONIC Center. The authors acknowledge C. Dietz and Y. K. Joshi from Georgia Institute of Technology for providing samples of cupric hydroxide superhydrophobic surfaces.
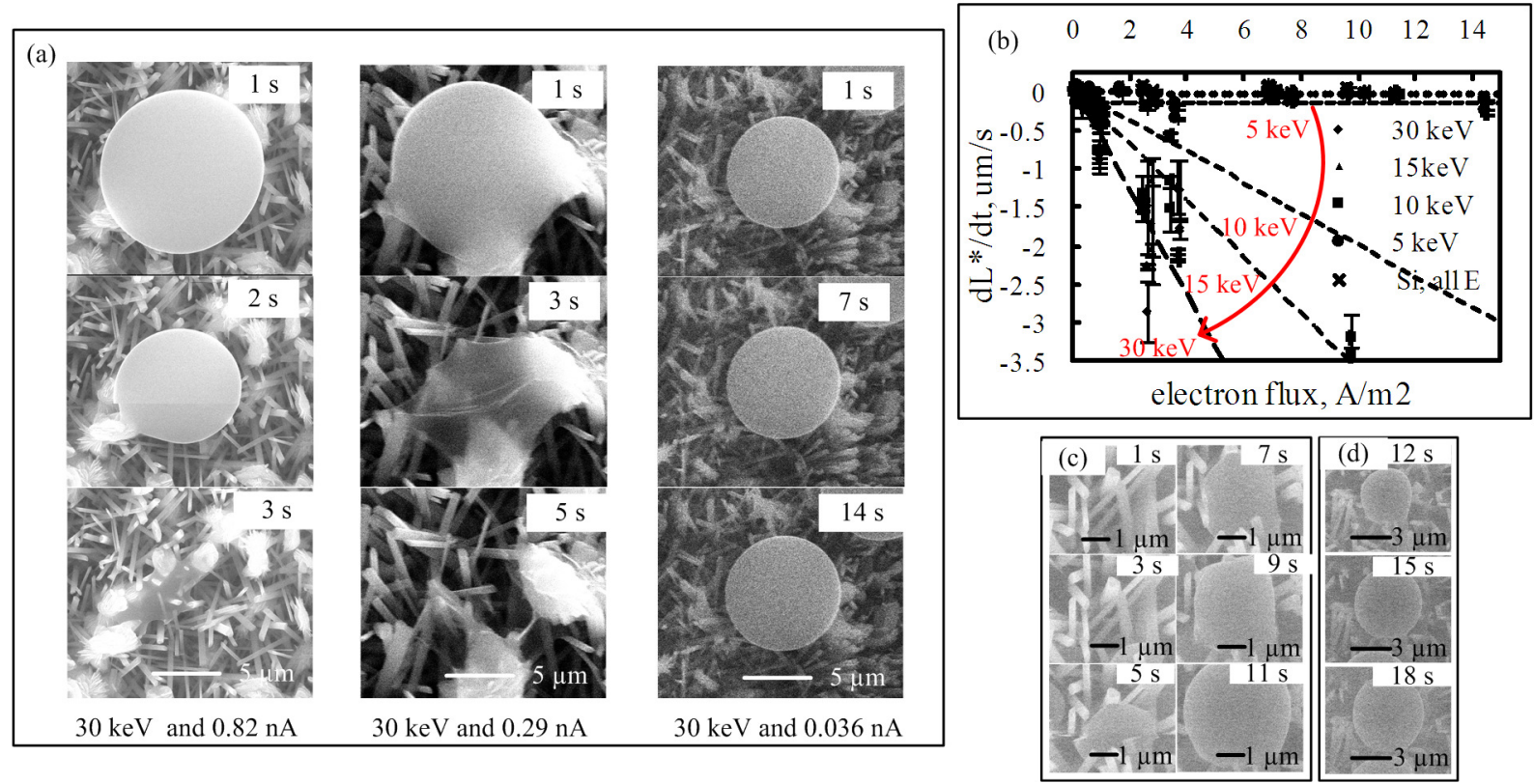

FIG. 1. (a) ESEM images of condensation on cupric hydroxide nanotube SHS at $930 \mathrm{~Pa}, 5^{\circ} \mathrm{C}, 20^{\circ}$ tilt, $6.2 \mathrm{~mm}$ working distance, $18.6 \mu \mathrm{m}$ by $17.1 \mu \mathrm{m}$ viewing area with indicated electron beam settings, (b) the rate of change of the characteristic length, $\mathrm{dL} * / \mathrm{dt}$, as a function of electron flux $\mathrm{F}$ (current/viewing area) for drops on a superhydrophobic surface at $20^{\circ}$ tilt for electron beam energies of $5 \mathrm{keV}, 10 \mathrm{keV}, 15 \mathrm{keV}$, and $30 \mathrm{keV}$ and on silicon surface at $\sim 54^{\circ}$ tilt for electron beam energies of $10 \mathrm{keV}, 15 \mathrm{keV}$, and $30 \mathrm{keV}$, (c-d) ESEM imaging of subsequent steps of single water drop growth process with a (c) $4.6 \mu \mathrm{m}$ by $4.3 \mu \mathrm{m}$, and (d) $9.3 \mu \mathrm{m}$ by $8.5 \mu \mathrm{m}$ viewing area imaged at 10 $\mathrm{keV}$ and $0.013 \mathrm{nA}$. 